\title{
Capacity of Interference-Limited Ad Hoc Networks with Infrastructure Support
}

\author{
Jeong-woo Cho, Student Member, IEEE, Seong-Lyun Kim, Member, IEEE, and Song Chong, Member, IEEE
}

\begin{abstract}
In this letter, we consider the capacity of ad hoc networks with infrastructure support. Although GrossglauserTse mobile network model enables $\Theta(1)$ per-node throughput scaling, the mobility assumption may be too unrealistic to be accepted in some practical situations. One of the key observations we acquired is that the infrastructure support plays the same role played by the mobility in the Grossglauser-Tse model. We show that nodes can utilize the randomly located infrastructure support instead of mobility when nodes are nearly static. In this case, we show that the per-node throughput of $\Theta(1)$ is still achievable when the number of access points grows linearly with respect to the number of nodes.
\end{abstract}

Index Terms-Ad hoc wireless network, throughput capacity, infrastructure support, mobility

\section{INTRODUCTION}

A Key concern in ad hoc networks is the throughput scaling law that may depend on many aspects of networks such as power control, scheduling strategies, routing schemes, network topology, and physical characteristics.

In their seminal paper [1], Gupta and Kumar showed that per-node throughput of $\Theta(1 / \sqrt{n \log n})$ is attainable assuming that $n$ number of mobile nodes are placed randomly. Roughly speaking ${ }^{\S}, f(n)=\Theta(g(n))$ means that $f(n)$ and $g(n)$ are of the same rate of growth. This result is rather pessimistic since per-node throughput should decrease very fast as the number of mobile nodes increases. In the subsequent paper [2], Grossglauser and Tse showed that per-node throughput of $\Theta(1)$ is achievable when mobility is fully exploited. Although this result seems to be optimistic at the first glance, in some realistic situations, nodes are nearly static. Consequently, delays experienced by source-destination pairs will be intolerable [3] and the throughput per node becomes very small. However, we still think that it is possible to achieve the capacity of $\Theta(1)$ by exploiting infrastructure support, even if the nodes are static. In this letter, the mobility is considered not as an essential component but as an additional component that can potentially increase the throughput of ad hoc networks.

Regarding the throughput capacity of ad hoc networks with infrastructure support, there have been several works [4]-[6]. Note that these works adopted the protocol model [1] for their

This work was supported by the MIC (Ministry of Information and Communication), Korea, under the grant for BrOMA-ITRC program supervised by IITA (Institute of Information Technology Assessment). Jeong-woo Cho and Song Chong are with Korea Advanced Institute of Science and Technology (KAIST), Daejon 305-701, Korea (email: ggumdol@netsys.kaist.ac.kr; song@ee.kaist.ac.kr). Seong-Lyun Kim is with Yonsei University, Seoul 120749, Korea (email: slkim@yonsei.ac.kr).

F Formally, $f(n)=\Theta(g(n))$ if there are constants $c_{1}>0, c_{2}>0, n_{0}$ such that $\forall n>n_{0}$ it is true that $c_{1} g(n)<f(n)<c_{2} g(n)$. interference model rather than the physical model [2], which is our case. Analytical results based on the protocol model may overestimate the capacity of ad hoc networks since they do not take interference from other users into consideration. This letter reveals that the per-node throughput of $\Theta(1)$ is also attainable even though the physical model is employed.

\section{NETWORK MODEL}

We consider a random network with infrastructure support where mobility can be potentially exploited. There are two tiers where infra tier is an infrastructure network composed of access points and ad hoc tier is composed of nodes. All access points and nodes are randomly located in the disk of unit area (of radius $1 / \sqrt{\pi}$ ). To make our model mathematically tractable, the bandwidth between any two access points is assumed to be relatively enough.

There are $n$ nodes in the ad hoc tier. For each slotted time $t$, we randomly designate $n_{S}=\theta n$ of the nodes as source nodes and the remaining $n_{R}=(1-\theta) n$ nodes as destination nodes. The source density parameter $\theta$ falls within the open interval $(0,1)$. Similar to [2], a destination node can be also a relay node. Since the designation changes at every time slot, the role of each node is varying. The number of access points $n_{A}$ is assumed to be proportional to $n$, i.e., $n_{A}=\psi n$ where $\psi$ is the infrastructure density parameter falling within $[0,1]$. The ad hoc tier model used in this letter is not very different from the one employed in [2].

In the ad hoc tier, a source node or relay node sends data to other nodes at $R$ bits/s through a single common channel. Each access point has $K(>0)$ uplink channels where each channel has the bandwidth of $R$ bits/s. We assume the full orthogonality among $K$ channels such that the transmissions occurring within one channel do not interfere with the other channels. We know that downlink traffic may not be evenly distributed to all access points but may be concentrated at a specified access point. For simplicity, we assume that downlink bandwidth is relatively abundant compared with uplink bandwidth.

\section{A. Interference Model}

For the interference model, we adopt a physical model where the main features of ad hoc networks are specified with the signal power of a node and the interference signal from other nodes. At time slot $t$, let $P_{i}(t)$ be the transmission power of node $i$ and $\gamma_{i j}(t)$ be the channel gain from node $i$ to node (or access point) $j$, such that the received power at $j$ is 


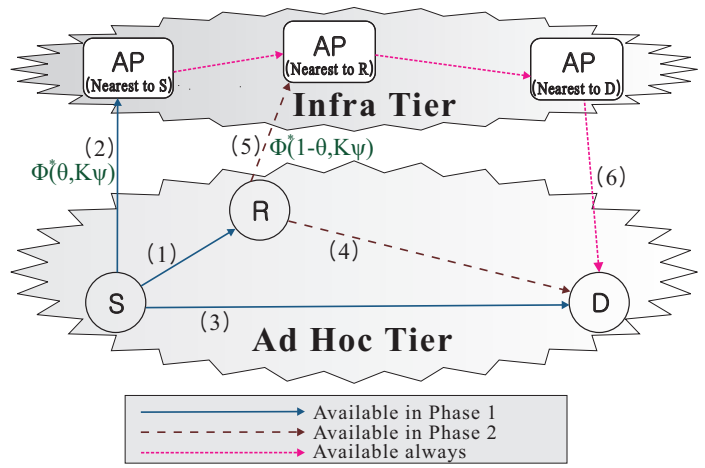

Fig. 1. The proposed scheduling policy.

$P_{i}(t) \gamma_{i j}$. The transmission from $i$ to $j$ at rate $R$ bits/s through channel $c$ is successful if

$$
\frac{P_{i}(t) \gamma_{i j}(t)}{N_{0}+\frac{1}{L} \sum_{k \in S_{c}, k \neq i} P_{k}(t) \gamma_{k j}(t)} \geq \beta
$$

where $S_{c}$ is the set of senders transmitting through channel $c, \beta$ is the signal-to-interference ratio (SIR) requirement for successful communication, $N_{0}$ is the background noise power, and $L$ is the processing gain of the system. The channel gain is assumed to be

$$
\gamma_{i j}(t) \stackrel{\text { def }}{=} \frac{1}{\left|X_{i}(t)-X_{j}(t)\right|^{\alpha}}
$$

where $\alpha$ is the path loss exponent greater than 2 and $\left\{X_{i}(t)\right\}$ is the location of node $i$ at time slot $t$.

\section{B. Proposed Scheduling Policy}

For the moment, we assume that the node location processes $\left\{X_{i}(t)\right\}$ are independent, stationary, and ergodic. The sets of source nodes, destination nodes, access points are denoted by $\mathcal{S}, \mathcal{R}$ and $\mathcal{A}$, respectively. Destination nodes also play the role of potential receivers, i.e., relay nodes. Thus nodes in $\mathcal{R}$ will be also called relay nodes. Let us focus on a certain time slot $t$ and its subsequent time slot $t+1$. There are two phases in scheduling policy $\pi$ as shown in Fig. 1 .

Phase I (Source Nodes Transmit): Each source node S transmits packets to its nearest relay node $\mathrm{R}$ through the single common channel (arrow (1) in Fig. 1) and transmits packets to its nearest access point AP through one of $K$ uplink channels (arrow (2) in Fig. 1) simultaneously. With very low probability of $\Theta(1 / n)$, the nearest relay node $\mathrm{R}$ can be the true destination $\mathrm{D}$ of the source node (arrow (3) in Fig. 1).

Phase II (Relay Nodes Transmit): Each relay node R transmits packets to its nearest relay node only if the relay node $\mathrm{R}$ has packets whose true destination $\mathrm{D}$ is the nearest relay node (arrow (4) in Fig. 1). Concurrently, each relay node $\mathrm{R}$ transmits packets to its nearest access point AP through $K$ uplink channels (arrow (5) in Fig. 1) in a random manner.

These two phases are interleaved: In the odd time-slots, Phase I is run. In the even time-slots, Phase II is run. Once an access point receives packets, the packets are assumed to be delivered to the access point which is nearest to the true destination within a brief instant. Then the access point transmits them to the true destination (arrow (6) in Fig. 1).
For simplicity, two kinds of mode are considered: In pure ad hoc mode, arrows (1), (3) and (4) are used. In pure infra mode, arrows (1), (2), (5), (6). It is already proven that $\Theta(1)$ per-node throughput is attainable in pure ad hod mode with mobility support [2].

\section{CAPACITY IMPROVEMENT WITH INFRASTRUCTURE SUPPORT}

Theorem 1 shows that there are $\Theta(n)$ feasible S-D pairs in pure infra mode, that is to say, per-node throughput of $\Theta(1)$ is attainable without mobility support.

Theorem 1: For ad hoc networks with scheduling policy $\pi$ and the identical transmission power of $p \neq O\left(n^{-\alpha / 2}\right)^{\boldsymbol{\pi}, \dagger \dagger}$, the expected number $\mathrm{E}\left\{N_{t}\right\}$ of feasible source-access point pairs in Phase $\mathrm{I}$ is $\Theta(n)$, i.e.,

$$
\lim _{n \rightarrow \infty} \frac{\mathrm{E}\left\{N_{t}\right\}}{n}=\Phi^{*}(\theta, K \psi)>0
$$

where the probability $\Phi^{*}(\theta, K \psi)$ is defined in Eq. (9). Furthermore, the expected number $\mathrm{E}\left\{N_{t}\right\}$ of feasible relay-access point pairs in Phase II is $\Theta(n)$, i.e.,

$$
\lim _{n \rightarrow \infty} \frac{\mathrm{E}\left\{N_{t}\right\}}{n}=\Phi^{*}(1-\theta, K \psi)>0 .
$$

(Note that the probability (9) is independent of $n$.)

Proof: We consider a fixed time slot $t$. Let $U_{1}, \ldots, U_{n_{S}}$ be the random positions of the sources in $\mathcal{S}$. Let $V_{1}, \ldots, V_{k}$ be the positions of access points in $\mathcal{A}$ where $k=\psi n$. These random variables are i.i.d. uniformly distributed on the open disk of unit area. For each node $s \in \mathcal{S}$, let its intended access points $a(s) \in \mathcal{A}$ be the access point that is nearest to $s$ among all access points in $\mathcal{A}$. Since there are $K$ uplink channels and source node $s$ chooses one channel randomly, without loss of generality, we assume that channel 1 is chosen by $s$.

We now analyze the probability of successful transmission for each source-access point pair. By symmetry, we can just focus on one such pair, say $(1, a(1))$. The event of successful transmission depends on the positions $U_{1}, \ldots, U_{n_{S}}$ and $V_{1}, \ldots$, $V_{k}$. Let $Q_{i}$ be the received power from source node $i$ at access point $a(1)$, and

$$
Q_{i}=p\left|U_{i}-V_{a(1)}\right|^{-\alpha}
$$

The node $a(1)$ satisfies

$$
a(1)=\underset{j \in \mathcal{A}}{\arg \min } p\left|U_{1}-V_{j}\right| .
$$

The total interference at access point $a(1)$ is given by $I=$ $\sum_{i \neq 1} Q_{i}$. The SIR for the transmission from sender 1 at access point $a(1)$ is given by

$$
\mathrm{SIR}=\frac{Q_{1}}{N_{0}+\frac{1}{L} I}
$$

\section{Step 1. Finding the Asymptotic Distribution of $Q_{1}$}

"Roughly, $f(n)=O(g(n))$ means that $f(n)$ does not grows faster than $g(n)$. Thus, $f(n) \neq O(g(n))$ means that $f(n)$ grows faster than $g(n)$.

${ }^{\dagger \dagger}$ For the case $p=\Theta\left(n^{-\alpha / 2}\right)$, Eqs. (2) and (3) are also satisfied. We do not deal with this case for brevity. 
We now analyze the asymptotics of $Q_{1}$ and $I$ as $n \rightarrow \infty$. Because $p$ is independent of $j, Q_{1}$ becomes

$$
Q_{1}=\max _{j \in \mathcal{A}} Z_{j}
$$

where $Z_{j}=p\left|U_{1}-V_{j}\right|^{-\alpha}$. Following an analogous procedure used in [2], one can show that

$$
\frac{1}{p(\pi \psi n)^{\alpha / 2}} Q_{1} \rightarrow Q_{\alpha}^{*}
$$

where $Q_{\alpha}^{*}$ has a cdf

$$
F_{Q_{\alpha}^{*}}(x)= \begin{cases}\exp \left(-x^{-2 / \alpha}\right), & x \geq 0 \\ 0, & x<0 .\end{cases}
$$

\section{Step 2. Finding the Asymptotic Distribution of I}

Since each source randomly chooses a channel among $K$ uplink channels between the source and its nearest access point, the total interference in a channel becomes a random sum of random variables $Q_{i}$. Since the probability that a sender chooses channel 1 is $1 / K$, the total inference experienced by $s$ becomes $I=\sum_{i \in S_{1}, i \neq 1} Q_{i}$ where $S_{1}$ denotes the set of sources that transmit data through channel 1 . Then the cardinality of $S_{1}$ has the following binomial distribution.

$$
\operatorname{Pr}\left\{\left|S_{1}\right|=m\right\}=\left(\begin{array}{c}
n \\
m
\end{array}\right)\left(\frac{1}{K}\right)^{m}\left(1-\frac{1}{K}\right)^{n-m}
$$

To rearrange $I$ into a sum of a deterministic number of random variables, we now derive the generating function $\mathbf{E}\left\{s^{I}\right\}$ of $I$ (See, e.g., [7]). Using the fact that the generating function of a random sum is a compound function [8, pp. 287], $\mathbf{E}\left\{s^{I}\right\}$ becomes

$$
\mathbf{E}\left\{s^{I}\right\}=\left(\frac{1}{K} \mathbf{E}\left\{s^{Q_{i}}\right\}+1-\frac{1}{K}\right)^{n} .
$$

Therefore, we can regard $I$ as a sum of $n_{S}-1$ number of $Q_{i}^{+}$ which has a cdf

$$
F_{Q_{i}^{+}}(x)= \begin{cases}1-\frac{1}{K}+\frac{1}{K} F_{Q_{i}}(x), & x>0 \\ 0, & x \leq 0 .\end{cases}
$$

Following an analogous procedure used in [2], one can show that

$$
\frac{1}{p[\pi \Gamma(1-2 / \alpha)(\theta n-1) / K]^{\alpha / 2}} I \rightarrow I_{\alpha}^{*}
$$

where $\Gamma(s)=\int_{0}^{\infty} x^{s-1} e^{-x} d x$, and $I_{\alpha}^{*}$ is the stable distribution defined in [9].

\section{Step 3. Asymptotic Independence of $\mathrm{Q}_{1}$ and $\mathrm{I}$}

Finally, we can easily show that $I$ and $Q_{1}$ are asymptotically independent following an analogous procedure used in [2]. Combining this last fact with Eqs. (4) and (6), we get the result on the probability of successful transmission from source node 1 to access point $a(1)$.

$$
\begin{aligned}
& \lim _{n \rightarrow \infty} \operatorname{Pr}\{\operatorname{SIR} \geq \beta\}=\lim _{n \rightarrow \infty} \operatorname{Pr}\left\{\frac{Q_{1}}{N_{0}+\frac{1}{L} I} \geq \beta\right\} \\
& \quad=\lim _{n \rightarrow \infty} \operatorname{Pr}\left\{\frac{p(\pi \psi n)^{\alpha / 2} Q_{\alpha}^{*}}{N_{0}+\frac{1}{L} p[\pi \Gamma(1-2 / \alpha)(\theta n-1) / K]^{\alpha / 2} I_{\alpha}^{*}} \geq \beta\right\}
\end{aligned}
$$

Furthermore, If $p \neq O\left(n^{-\alpha / 2}\right)$, that is, $p$ grows faster than $n^{-\alpha / 2}$ (e.g., $p$ is a constant or $p=\Theta\left((\log n / n)^{\alpha / 2}\right)$, then $n^{\alpha / 2}$ terms are canceled out and the Eq. (7) becomes

$$
\operatorname{Pr}\left\{\frac{Q_{\alpha}^{*}}{I_{\alpha}^{*}} \geq \frac{\beta}{L}\left[\frac{\theta}{K \psi} \Gamma\left(1-\frac{2}{\alpha}\right)\right]^{\alpha / 2}\right\}
$$

which is independent of $n$. Thus Eq. (2) is satisfied with

$$
\Phi^{*}(\theta, K \psi) \stackrel{\text { def }}{=} \theta \cdot \operatorname{Pr}\left\{\frac{Q_{\alpha}^{*}}{I_{\alpha}^{*}} \geq \frac{\beta}{L}\left[\frac{\theta}{K \psi} \Gamma\left(1-\frac{2}{\alpha}\right)\right]^{\alpha / 2}\right\}
$$

and the expected number of feasible source-access point pairs is $\Theta(n)$. Similary, we can verify Eq. (3) easily.

The probabilities of successful transmission $\Phi^{*}(\theta, K \psi)$ and $\Phi^{*}(\theta, K \psi)$ are independent of $n$ and strictly positive. Thus, the above theorem says that the expected numbers of sourceaccess point pairs and relay-access point pairs grow linearly with respect to the number of nodes $n$ if the number of access points grows linearly with the number of nodes.

Access points in pure infra mode play the same role played by relay nodes in pure ad hoc mode. Thus, similar to [2], we have proved the following theorem.

Theorem 2: In pure infra mode, each node achieves throughput of $\Theta(1)$.

\section{CONCLUSION}

We have proved that the per-node throughput of ad hoc networks with infrastructure support is still $\Theta(1)$ based on the physical model, which captures essential characteristics of interference-limited ad hoc networks.

The transmission power requirement that, $p$ can be decreased on the order of $\frac{1}{n^{\alpha / 2}}$ as $n$ grows, is somewhat surprising at a first glance. However, note that it is unrealistic since the channel gain $\gamma_{i j}$ in Eq. (1) is invalid when two nodes are very close [10]. Thus, we note that the adoption of more accurate physical model is crucial for establishing more genuine per-node throughput in ad hoc wireless networks.

\section{REFERENCES}

[1] P. Gupta and P. R. Kumar, "The capacity of wireless networks," IEEE Trans. Information Theory, vol. 46, no. 2, pp. 388-404, Mar. 2000

[2] M. Grossglauser and D. N. C. Tse, "Mobility increases the capacity of ad hoc wireless networks," IEEE/ACM Trans. Networking, vol. 10, no. 4, pp. 477-486, 2002.

[3] A. E. Gamal, J. Mammen, B. Prabhakar, and D. Shah, "Throughputdelay trade-off in wireless networks," in Proc. of IEEE INFOCOM, 2004

[4] B. Liu, Z. Liu, and D. Towsley, "On the capacity of hybrid wireless networks," in Proc. of IEEE INFOCOM, 2003.

[5] U. C. Kozat and L. Tassiulas, "Throughput capacity of random ad hoc networks with infrastructure support," in Proc. of ACM MOBICOM, 2003.

[6] A. Zemlianov and G. D. Veciana, "Capacity of ad hoc wireless networks with infrastructure support," IEEE J. Sel. Areas Commun., vol. 23, no. 3, pp. 657-667, 2005.

[7] A. Papoulis and S. U. Pillai, Probability, Random Variables and Stochastic Processes, 4th ed. New York: McGraw-Hill, 2002.

[8] W. Feller, An Introduction to Probability and Its Applications, 3rd ed. New York: Wiley, 1968, vol. I.

[9] — An Introduction to Probability and Its Applications, 2nd ed. New York: Wiley, 1971, vol. II.

[10] O. Dousse and P. Thiran, "Connectivity vs capacity in dense ad hoc networks," in Proc. of IEEE INFOCOM, 2004. 